\title{
The incidence of depression among residents of assisted living: prevalence and related risk factors
}

\author{
This article was published in the following Dove Press journal: \\ Clinical Interventions in Aging \\ 9 October 2017 \\ Number of times this article has been viewed
}

\author{
Fidaa M Almomani' \\ Wegdan Bani-issa ${ }^{2}$ \\ 'Department of Rehabilitation \\ Sciences, Faculty of Applied Medical \\ Sciences, Jordan University of \\ Science and Technology, Irbid, Jordan; \\ ${ }^{2}$ Department of Nursing, College of \\ Health Sciences, University of Sharjah, \\ Sharjah, United Arab Emirates
}

Correspondence: Fidaa M Almomani

Department of Rehabilitation Sciences, Faculty of Applied Medical Sciences, Jordan University of Science and Technology, P.O. Box No 221 I0, Irbid, Jordan

Tel +9627201000

Fax +9622701100

Email falmomani@just.edu.jo
Aim: This study aims to recognize and estimate the prevalence of depression and its risk factors among residents of assisted living facilities (ALs) in Jordan.

Background: Depression is commonly experienced by residents of ALs. The condition is, however, often misunderstood as a part of normal aging and may be overlooked by health care professionals. Little is known about the extent of depression and its risk factors among AL residents in Jordan.

Subjects and methods: A national representative sample of 221 residents selected from all AL units across Jordan was recruited to the study. Data on expected risk factors for depression were collected, including sociodemographics; smoking status; number of roommates; number of family members; assessments for cognitive functioning, for lower limb functioning, for hand, shoulder, and arm impairments; and oral health status. Levels of depression among the sample respondents were also assessed.

Results: The study found that around $60 \%$ of the participants reported depressive manifestations, with $48.0 \%$ of AL residents exhibiting impaired cognitive functions, one-third $(33.2 \%)$ having $>50 \%$ upper limb disability, two-thirds (63.2\%) being at moderate risk of falls, and $69.7 \%$ having fair to poor oral health status. Being female, and having a higher level of education, disability of the upper limbs, and impairment of cognitive functions were found to be independent risk factors for depression in participants.

Conclusion: Depression is relatively common among residents of AL units in Jordan. Health care professionals, nurses, physiotherapists, and dentists working in these facilities need to work cooperatively to identify the manifestations of depression in residents and collaboratively implement the best practice in the treatment of depression and circumvent its long-term impacts on the health of residents.

Keywords: depression, assisted living, risk factors, cognitive disabilities, physical disabilities, oral health

\section{Introduction}

Depression is a common occurrence in later life, affecting $7 \%$ of the general elderly population. ${ }^{1}$ A decline in physical, mental, and cognitive abilities, coupled with retirement and the loss of contact with social networks, and the higher likelihood of deaths among loved ones are factors that contribute to the development of depression in older adults. $^{2-7}$ Symptoms such as persistent sadness, anxiety, feelings of hopelessness, fatigue, low energy, suicidal thoughts, insomnia, and aches and pains may all be indicators of depression in later age. ${ }^{8}$

Evidence exists in the literature for the significant prevalence of depression among older population living in assisted living facilities (ALs). Life inside ALs can be challenging for residents and could place them at greater risk for depression compared 
to those individuals who are living in their own homes in the community. ${ }^{8-13}$ A national survey to assess depression among long-term AL residents in the USA reported a high rate of depression among this population during the first year of placement, which was mainly attributed to pain, poor physical health, and prior institutionalization. ${ }^{14}$ Similarly, Samus et al found that three-quarters of residents $(n=200)$ randomly selected from 21 ALs in the USA suffered from depression. The authors of that study called for early recognition, screening, and treatment of depression and psychiatric symptoms in residents. ${ }^{15}$

Depression may be the main cause of disturbed sleep, poor eating habits, impaired physical activity and cognitive and social functioning, and exacerbated self-neglect, symptoms which, in turn, are associated with increased mortality. ${ }^{10,12,16-18}$ Nevertheless, there is a common lack of both recognition of and screening for depression among AL residents; the main medical focus on the elderly concerns their physical health. ${ }^{8,19}$ Therefore, depression is often left untreated, and this may result in further negative consequences. ${ }^{10,12,17,18}$

Risk factors that place residents at risk for depression vary across AL populations and cultures. ${ }^{14} \mathrm{~A}$ cross-sectional study from Iran, for example, found that $10.7 \%$ of residents of ALs $(n=244)$ experienced severe depression, and that those who were less satisfied with their personal lives, unhappy with the quality of food offered, and less active had a greater likelihood of being severely depressed compared to other residents. In Norway, another cross-sectional study found that having a sense of "meaning in life" contributed to better psychologic health in AL residents. ${ }^{20}$ Another study, also from Norway, by Barca et al found that elderly people who were not married, had shorter periods of AL residence, and were taking antidepressant medication were at greater risk of depression compared to other residents. ${ }^{21}$ Other studies have reported different risk factors for the development of depression, such as the level of social engagement and experience of belonging in AL communities. ${ }^{11,22}$

It is neither known whether a similarly high prevalence of depression exists among AL residents in other countries, such as Jordan, nor its risk factors. Because the concept of AL is relatively new in Jordan, there are no standards for the structure, living arrangements, or type and quality of care provided. ${ }^{23,24}$ Assisted living care is provided by staff who are neither adequately prepared nor qualified to manage the needs of older people specifically. The main focus of care for AL residents in Jordan is on providing basic needs such as meals, bathing and other personal care activities, medication assistance, and housekeeping. The quality and range of services provided depend on the number of staff and the level and nature of residents' disabilities and needs - physical, psychologic, and mental. ${ }^{23,24}$

The primary objective of this study is to investigate the prevalence of depression and its risk factors in AL residents. Therefore, it aimed to assess the incidence of depression symptoms among AL residents in Jordan and to ascertain the risk factors for the development of depression. Unlike other studies, our study includes a comprehensive examination of possible risk factors for depression, including sociodemographic, smoking habits, cognitive status, balance and gait, impairment of arm, shoulder, and hand function, and oral health status. Because of its often devastating consequences, depression among AL residents needs to be addressed by health care providers.

The main objective of the study is to assist in the development of targeted and culturally appropriate interventions that will allow for early detection, screening, and treatment of depression, and consequent improvement in the quality of life and daily activities of individuals living in Jordanian AL homes.

Research questions are as follows:

1. What is the incidence of depression in older adults living in AL in Jordan?

2. What are the risk factors for developing depression in older adults living in AL in Jordan?

\section{Subjects and methods Design}

This cross-sectional study uses a descriptive and correlational design to investigate the incidence of depressive symptoms among AL residents in Jordan and the independent risk factors for their developing depression.

After obtaining ethical approval, the principal investigator contacted the concerned AL administrations to gain access to the site. This was followed by visiting the site by the research team to recruit subjects. In the first visit, the research team gathered older adults with the presence of administration to explain the study, requested their participation, and took written informed consent from them. Data collection expanded over a 3-month period.

\section{Study settings and recruitment of subjects}

Jordan has $11 \mathrm{AL}$ facilities distributed in the north and center of the country, housing a total number of $\sim 297$ residents with a mean age of 60 years. All residents were recruited to participate in this study. Consent for participation was approved 
by the institutional review board at King Abdullah University Hospital and faculty of research at Jordan University of Science and Technology/Irbid/Jordan. All participants signed the consent form before data collection. All signed consents by the participants were informed. Subjects with mental retardation, severe dementia, or whose responses would lack validity were excluded from the study.

Permission was obtained from subjects for their medical histories (medications, previous hospitalizations, chronic conditions, and so on) to be accessed from the subjects' medical files held at their respective AL unit.

A trained research assistant collected demographic information from the subjects and their files. These data included date of birth and gender, marital status, level of education and previous occupation, amount of monthly income where applicable, and their smoking habits.

\section{Instruments and tools}

A qualified and trained research therapist performed the following evaluations and assessments: Geriatric Depression Scale (GDS), ${ }^{25}$ Mini-Mental State Examination (MMSE), ${ }^{26,27}$ the Tinetti Assessment Battery for gait and balance (TAB), ${ }^{28}$ and the Disability of Arm, Shoulder, and Hand assessment (DASH). ${ }^{29}$ Oral health status was evaluated by a certified dentist using the Arabic version of the Geriatric Oral Health Assessment Index. ${ }^{30}$

The MMSE is a simple tool that is used to evaluate the degree of cognitive impairment. There is a short version (10 minutes, 30 items) used in this study and a longer version (90 minutes, 120 items) of this tool. The short version consists of simple questions about a person's orientation to place and time, registration of prompts, questions about language use (naming) and comprehension, arithmetic patterns, and testing of spatial skills (such as in drawing). ${ }^{26,27}$ The total score achievable is 30 , with a score of 26 or greater indicating normal cognitive functioning. ${ }^{26}$

The GDS is a short (30-item), simple (yes/no question) self-report assessment that is commonly used to evaluate the level of depression among an elderly population. Its very good-excellent psychometric properties and simplicity of administration enable the scale to be used for individuals with mild-moderate cognitive impairments. The scores are interpreted as normal (0-4), mild depression (5-8), moderate depression (9-11), and severe depression (12-15). ${ }^{25}$ In this study, a score of 10 or more indicated a subject with depression.

The TAB is a performance-oriented tool with excellent reliability used to measure the gait (12 points) and balance
(16 points) of an individual performing specific tasks, for a maximum achievable score of 28. For instance, the person is asked to stand on one foot (eyes opened or closed), stand up from a chair, walk for 10 steps, and so on. Each task is scored between 0 and 2 , with a 0 score indicating maximum impairment and a score of 2 indicating no impairment. In this study, a total score of $<19$ indicated individuals with impairment in gait and balance. ${ }^{28}$

The DASH is a self-reporting 30-item questionnaire with excellent psychometric properties used to assess impairment and pain in the joints of the arm, shoulder, and hand on a scale from 0 (no impairment) to 100 (maximum impairment) of the upper limbs. ${ }^{29}$

The Geriatric Oral Health Assessment Index is a 12-item self-report dental health questionnaire developed for use in elderly populations. Each question is scored between 1 and 5, with the cumulative score from 12 questions representing a minimum to maximum range from 12 to 60 points. Individuals are asked general questions related to their perceptions of oral health relating, for instance, to pain and discomfort in the mouth, whether they need dental/oral treatment, and whether they are satisfied with their dental condition, their assessment of the requirement for treatment, and the tolerability of oral pain and discomfort. The Arabic translation of the instrument has excellent reliability (Cronbach's alpha $=0.93$ ) and very good validity (correlation coefficient $>0.70$ ). ${ }^{30}$

As this study sample included many illiterate subjects, all assessments and questionnaires were completed in personal interviews conducted by trained research assistants.

\section{Translation procedure and pilot testing}

The DASH, the TAB, the MMSE, and the GOHAI have Arabic language applications. The adequacy of the psychometric properties of the Arabic versions of these instruments has been validated in previous research: $\mathrm{DASH},{ }^{29,31} \mathrm{TAB},{ }^{2}$ MMSE, ${ }^{32}$ and GOHAI. ${ }^{30}$

Further, a forward-and-backward translation method was used to translate the GDS into Arabic language. ${ }^{33}$ The forward translation of the GDS was performed by four bilingual researchers (associate professors). Differences of opinion in the translation of specific terms were resolved by discussion until agreement was reached by consensus. The backward translation of the instrument was made by a native English speaker who could speak Arabic fluently and was not familiar with the original English version of this instrument. Eleven researchers then evaluated the extent of similarity between the forward- and the backward-translated items. The similarity of linguistic forms and meanings were evaluated. 
Similarity scores ranged from 0 (not similar) to 1 (similar). A score of 0.8 or higher indicated that $80 \%$ of the evaluators agreed on the translation. All items scored between 0.8 and 1 with adequate reliability of the Arabic translated version of the instrument (Cronbach's alpha coefficient $=0.84$ ). The initial versions of the instrument were pilot-tested on a group of 20 study participants and revised accordingly by the researchers.

\section{Data processing and analysis}

The study data were saved and analyzed using the SPSS ${ }^{\circledR}$ software (version 20). The significance level was set as $P \leq 0.05$. Odds ratios were calculated with $95 \%$ CIs. Cronbach's alpha coefficient was calculated for the translated GDS. Mean values, SDs, and frequency distributions were calculated for all study variables. Multiple logistic regression was performed to predict potential factors related to outcome variable (a score of $\geq 10$ in the GDS). The set of independent variables consisted of age, gender, marital status, education, previous occupation, income, smoking, number of roommates, number of family members, DASH score, TAB score, MMSE score, and GOHAI score. The main dependent variable was depression (a score of $\geq 10$ in the GDS considered as depressed participant).

\section{Results \\ Overview}

The study found that individuals in AL facilities in Jordan exhibited marked deterioration of physical and cognitive abilities, which were significantly correlated with manifestations of depression.

\section{Response rate, sites, and general description of participants}

Of the total 11 existing assisted living residences in Jordan, 3 refused their residents' participation in the study, citing privacy reasons. The number of residents in participating facilities was 297, which represented $72.7 \%$ of all AL residents in the final response rate. The exclusion of residents with severe dementia, mental retardation, or who refused to participate $(n=76)$ resulted in a final response rate of $74.4 \%$ of the total number of residents in participating AL units.

\section{Demographic profile}

The mean age of participants in this study was 62.4 (SD =13.9) years. Less than half of them $(45.2 \%)$ were female. More than half of the respondents $(53.4 \%)$ had a bachelor degree level of education and almost the same proportion was previously unemployed. About one-third of the participants were smokers and two-thirds of all participants had at least two medical conditions, with hypertension and diabetes being the most prevalent, followed by stroke and joint diseases (Table 1).

\section{Cognitive, physical, and mental functions} and oral health scores of the participants Approximately half of the sample had impaired cognitive capacities, as measured by the MMSE scores. Almost a third of the participants had $>50 \%$ upper limb impairment, as measured by the DASH assessment. Slightly less than two-thirds were found to be at high to moderate risk of falls, as measured

Table I Demographic and personal data of the study subjects $(\mathrm{N}=22 \mathrm{l})$

\begin{tabular}{|c|c|}
\hline Variable & n (\%) \\
\hline \multicolumn{2}{|l|}{ Age (years) } \\
\hline$<55$ & $69(31.2)$ \\
\hline $55-65$ & $69(31.2)$ \\
\hline$>65$ & $83(37.6)$ \\
\hline \multicolumn{2}{|l|}{ Gender } \\
\hline Male & I2I (54.8) \\
\hline \multicolumn{2}{|l|}{ Marital status } \\
\hline Single & II 8 (53.4) \\
\hline Married & $54(24.4)$ \\
\hline Widowed & $28(12.7)$ \\
\hline Divorced & $21(9.5)$ \\
\hline \multicolumn{2}{|l|}{ Educational level } \\
\hline Illiterate & $90(40.7)$ \\
\hline Primary/secondary education & $83(37.5)$ \\
\hline High school and above & $48(21.7)$ \\
\hline \multicolumn{2}{|l|}{ Previous occupation } \\
\hline Unemployed & II 4 (5I.6) \\
\hline Employed & $107(48.4)$ \\
\hline \multicolumn{2}{|c|}{ Monthly income (70 JD $=\$ 100)$} \\
\hline No income & $|5|(68.3)$ \\
\hline$\leq 100 \mathrm{JD}$ & $66(29.9)$ \\
\hline $101-250 \mathrm{JD}$ & $4(1.8)$ \\
\hline \multicolumn{2}{|l|}{ Smoking habit } \\
\hline No & $143(64.7)$ \\
\hline \multicolumn{2}{|c|}{ Number of diseases/medical conditions } \\
\hline $0-1$ & $87(39.4)$ \\
\hline 2 & $64(29.0)$ \\
\hline$\geq 3$ & $70(31.7)$ \\
\hline \multicolumn{2}{|c|}{ Type of diseases/medical conditions (yes) } \\
\hline Diabetes mellitus & $52(23.5)$ \\
\hline Hypertension & $74(33.5)$ \\
\hline Stroke & $38(17.2)$ \\
\hline Arthritis & $20(9.0)$ \\
\hline Joint disease & $38(17.2)$ \\
\hline Brain syndrome & $19(8.6)$ \\
\hline Psychiatric disorders & I0I (45.7) \\
\hline Gastrointestinal diseases & II (5.0) \\
\hline Renal diseases & $14(6.3)$ \\
\hline
\end{tabular}


Table 2 Descriptive statistics for MMSE score, DASH test score, TAB score, GOHAl score, and GDS ( $\mathrm{N}=22 \mathrm{I})$

\begin{tabular}{ll}
\hline Variable & $\mathbf{n}(\%)$ \\
\hline MMSE scores & \\
Impaired $(<26)$ & $105(47.7)$ \\
Not impaired $(\geq 26)$ & $115(52.3)$ \\
Mean (SD) & $21.76(9.5)$ \\
DASH scores & \\
$<50 \%$ & $147(66.8)$ \\
$\geq 50 \%$ & $73(33.2)$ \\
Mean (SD) & $32.89(30.6)$ \\
TAB scores & \\
$<23$ (high and moderate risk of fall) & $139(63.2)$ \\
$\geq 23$ (low risk of fall) & $81(36.8)$ \\
Mean (SD) & $15.6(10.9)$ \\
GDS scores & \\
(0-9) Normal & $89(40.5)$ \\
(I0-19) Mild depression & $62(28.2)$ \\
(20-30) Severe depression & $69(31.4)$ \\
Mean (SD) & $17.3(9.7)$ \\
GOHAl scores & \\
(I2-29) Poor & $87(39.4)$ \\
(30-39) Fair & $67(30.3)$ \\
(40-49) Good & $59(26.7)$ \\
(50-60) Very good & $8(3.6)$ \\
\hline A6brevition &
\end{tabular}

Abbreviations: DASH, Disability of Arm, Shoulder, and Hand assessment; GDS, Geriatric Depression Scale; GOHAI, Geriatric Oral Health Assessment Index; MMSE, Mini-Mental State Examination; TAB, Tinetti Assessment Battery.

by the TAB. Slightly more than two-thirds of the subjects were suffering from fair to poor oral health (Table 2).

Depressive symptoms were found to be experienced by around $60 \%$ of the study sample, as measured by the GDS, with $31.4 \%$ exhibiting severe depressive symptoms. Around $76 \%$ of the subjects also reported having trouble concentrating, $68 \%$ reported that they did not find life very exciting, and $>50 \%$ reported that they felt their life to be empty, were bored, had stopped many of their interests/activities, and did not feel hopeful about their future.

\section{Independent correlates of depression in participants}

Table 3 shows the independent correlates of depressive symptoms among the study subjects. A multivariate logistic regression model demonstrated the following predictive factors: age, assisted living, gender, marital status, education, previous occupation, number of roommates, average monthly income, number of family members, smoking, the DASH score, the TAB score, the MMSE score, the GOHAI score, and the number of medical diseases. The final model for risk of having depressive symptoms included the following predictors: assisted living, high school or above educational
Table 3 Results of multiple linear regressions: independent correlates of depression ( $\mathrm{N}=22 \mathrm{I})$

\begin{tabular}{|c|c|c|c|c|}
\hline \multirow{2}{*}{$\begin{array}{l}\text { Independent } \\
\text { variable }^{a}\end{array}$} & \multirow{2}{*}{$\frac{R=0.313}{B}$} & \multirow{2}{*}{$\frac{R^{2}=0.098}{t \text {-value }}$} & \multirow[t]{2}{*}{ Significance } & \multirow[t]{2}{*}{ Odds (Cl) } \\
\hline & & & & \\
\hline Gender & -3.636 & -2.446 & 0.015 & 4.7 \\
\hline Education & 1.536 & 2.352 & 0.020 & 3.7 \\
\hline MMSE score & 1.109 & -2.458 & 0.022 & 2.3 \\
\hline DASH score & 1.321 & 2.262 & 0.035 & 2.1 \\
\hline TAB score & -1.050 & -1.799 & 0.045 & 0.67 \\
\hline
\end{tabular}

Note: ${ }^{a}$ Nursing homes, age, gender, marital status, education, previous occupation, number of roommates, average monthly income, number of family members, smoking, DASH score, TAB score, MMSE score, GOHAl score, and number of medical diseases.

Abbreviations: DASH, Disability of Arm, Shoulder, and Hand assessment; GOHAI, Geriatric Oral Health Assessment Index; MMSE, Mini-Mental State Examination; TAB, Tinetti Assessment Battery.

level, upper limb disabilities (DASH $\geq 50 \%$ ), being female, and having impaired cognitive function (MMSE $<26$ ).

Residents with a higher educational level were about 3.7 times more likely to have symptoms of depression compared with residents who had lower level or no education. Female residents were about 4.7 times more likely to have symptoms of depression compared to males. Residents with upper limb disabilities of $>50 \%$ were about 2.1 times more likely to have symptoms of depression compared with those whose upper limb mobility had $<50 \%$ impairment. Furthermore, residents with cognitive impairment were 2.3 times more likely to have depressive symptoms than those whose cognitive functions were unimpaired.

In addition, the levels of depression in some assisted living residences were found to be greater than others, and the name/type of AL was found to be a factor in the incidence of depression in their residents. For privacy reasons, the results for depression in relation to the name of AL (ie, the best and the worst in terms of the prevalence of depression) will not be reported in this research.

\section{Discussion}

This study is the first to attempt to shed light on the incidence of depression in assisted living populations in Jordan. The prevalence of depression and its potential risk factors were estimated from assessments of sociodemographic variables, smoking habits, and cognitive, physical, and oral health status using standardized, valid, and reliable assessment tools. The comprehensiveness of assessments made using tools of established reliability and validity is one of the major strengths of the study.

Findings showed that AL residents suffered from impaired physical and cognitive functioning, manifesting as symptoms that are the risk factors for depression. Taking the degree of 
health impairments into consideration, these results have important implications for the health practitioners working in ALs, given the reported effects ${ }^{3,34-36}$ that poor physical, cognitive, and oral health functioning has on the independence and quality of life of residents. Further, decline in the health of AL residents affects their relationships and interactions in daily life, and is a cause of isolation, separation from others, loneliness, and more severe manifestations of depression. ${ }^{5,37}$

While in normal aging, the accumulation of chronic diseases and increased risk of functional decline and loss of independence are expected, research in the related literature has highlighted the importance of lifestyle factors in slowing the decline of physical and cognitive functions. Such studies have suggested that appropriate physical activity in the form of rehabilitative therapies and scheduled exercise may delay functional and cognitive impairment in AL residents. ${ }^{38}$ The significant deterioration in physical health found in this study sample represents a challenge to the health care system in Jordan that relates to the cost of treatment and the number of health professionals required to improve AL residents' quality of life. On this basis, the results of our study found assisted living conditions themselves to be a potential risk factor in the levels of depression experienced by residents. ALs in Jordan, as in other countries, vary in their structure, arrangements, and in the type and quality of care given to residents (eg, meals, housekeeping, personal care, medication assistance, and so on). In Jordan, there is a tendency to keep staffing levels in these facilities to a minimum. The results of this study demonstrate the necessity for a range of therapists and registered nurses, as well as dentists, to be employed to address AL residents' physical disabilities, problems with mobility, and exercise and oral health needs as a starting point to improve their health and well-being. ${ }^{31,17}$ Therapists would ideally need to understand the connection between cognitive function and the physical aspects of health, and its impact on the daily lives of AL residents. ${ }^{38}$

As expected, a significant number of participants in the study sample reported symptoms of depression. The results for its prevalence align with those from the literature that indicate a high incidence of depression symptoms in $\mathrm{AL}$ residents. ${ }^{12,15,39}$ Although depression is also considered to be an aspect of the aging process and is, therefore, generally not assessed in geriatric populations, ${ }^{40}$ the current best practice in the management of depression in AL residents is based on early screening and treatment. ${ }^{9,41}$ In Jordan and in the Middle East more widely, a stigma associated with mental illness is evident. ${ }^{42}$ In assisted living situations, health care providers do not screen for symptoms associated with depression, restricting their attention to physical illnesses, since this is considered more appropriate for the aged. ${ }^{40,43}$ The findings for the prevalence and impact of depression on AL residents' daily functioning in this study present a clear case for addressing their mental health. Routine screening for depression should be undertaken to enable a smooth transition to the AL environment. ${ }^{41,44}$ Furthermore, inadequate screening is a considerable barrier to better managing later-life depression in AL residents.

The best practice inside the AL environment has been reported to emphasize on the ability of staff to recognize signs of depression and make early interventions, for which training is essential. ${ }^{37}$ Previous research has found that residents in assisted care facilities suffering from depressive symptoms experience frustration with their careers' limited abilities to effectively deal with depression. ${ }^{9,45}$ Therefore, an interdisciplinary approach to raise awareness among staff for early recognition of the symptoms of depression among $\mathrm{AL}$ residents is recommended.

Apart from screening for depressive symptoms, it is also important that residents at risk for the condition are recognized. This may vary between cultures. In our study, female residents who were more educated and had upper limb disabilities and impaired cognitive function were found to be at greater risk for depression compared to others. In a similar study in Iran, no significant gender difference was found in reported symptoms, ${ }^{12}$ whereas in Canada, being female and having cognitive and physical impairments were identified as correlates of depression among AL residents. ${ }^{46}$ Similarly, Atkins et al found that Australian counterparts with functional limitations were at increased risk of developing distress and depression manifestations. ${ }^{47}$

This study found that the oral health status of our residents did not significantly correlate with depression, a result dissimilar to the findings of previous studies. ${ }^{3-5,17}$ Elsewhere, depression and other chronic mental illnesses correlate with poor oral health status. For example, a recent research by Watanabe et al on a group of 4,720 frail elderly ( $>65$ years) found that on the GDS, oral health and functioning were significantly associated with frailty. ${ }^{4}$ Similarly, Hybels et al, in a study of 944 older adults, found the symptoms for depression to be associated with poorer dental and oral health. ${ }^{3}$ In addition, the marital status of our residents did not correlate with depression, whereas in Norway, AL residents who were married showed higher levels of depression compared to single residents. ${ }^{21}$ Therefore, AL residents at risk for depression manifestation need to be identified upon admission to 
assisted living and followed up with close supervision of their psychologic health by the staff. Family involvement at the admission stage is also important to better guide health care professionals in their identification of residents at risk for depression through the introduction of screening and intervention options to manage depression. . $^{13,37}$

Interventions and rehabilitation programs targeting modifiable risk factors have the potential to prevent the development of depression. In particular, encouraging mobility and activities which stimulate cognitive functions may reduce psychologic distress and improve enjoyment of life..$^{41,44}$ These remedial measures will not, however, be effective in treating depression in AL residents in Jordan, unless the society changes its perception of people who have no alternative in later life to assisted living. It has been found that showing negative attitudes on the part of administrators and health care providers toward AL residents, coupled with a lack of cultural acceptance, remains one of the challenges to providing optimal care as well as for the acceptance of care by residents ${ }^{48}$ This evidence, and the results of this study, points to an urgent need to modify caring parameters and revise $\mathrm{AL}$ structures and procedures to integrate training for the recognition and treatment of mental health problems within these facilities. A new model for assisted living should encompass an interdisciplinary approach among health care professionals and be promoted across social networks and society.

Finally, this study found that AL facilities in Jordan do not cater exclusively for elderly people who cannot support themselves and have no familial supports; residents may include people younger than 55 years. The Jordanian government allows individuals with different physical or intellectual disabilities to be placed in assisted living residences. These individuals should also be assessed for depression, given they have no means of support outside the institution. The missions of AL institution in Jordan need to be recast to determine the criteria for admission, while providing alternative support for people who are still capable of functioning well in the community.

\section{Limitations}

While this study has highlighted the overlooked issue of depression among Jordan's assisted living population, and has found its independent correlates using standardized assessments in a nationally representative sample, other correlates, such as visual and hearing impairments, social supports, sense of meaning of life, and the period of $\mathrm{AL}$ residence, were not examined. Furthermore, the sample consisted of a wide age range and more than one-third of the participants were aged $<55$ years. Therefore, factors associated with depression in this study cannot be generalized to other AL environments with a majority of elderly population. That age was not found to be a significant predictor of depression is an interesting finding of this study. The correlates of depression presented might be important for $\mathrm{AL}$ residents of all ages. Another limitation of this study relates to the cutoff scores of the MMSE and GDS. Compared to other studies using different scores, the cutoff scores used here are more lenient. Thus, a higher number of individuals with lower levels of cognitive impairment or depressive symptoms may have been identified than in studies with higher cutoff scores. Moreover, in the analysis of the study data, multivariate linear and logistic regressions were used, which could increase the rates of type I error. Therefore, it is important to analyze the results of this study in light of it being the first endeavor to understand the prevalence of depression and the correlated risk factors among AL residents in Jordan. Additional research is required to confirm and refine the findings of this study.

\section{Conclusion}

Depression is a very common health problem of residents in AL facilities in Jordan, especially for females and better educated individuals with impaired upper limb and cognitive functioning. It is recommended that the structure and policy of assisted living institutions be revised to include routine assessment for symptoms of depression and better access to medical and rehabilitative care for residents to meet their multiple health needs. ${ }^{49,50}$

Programs to treat and mitigate the incidence of depression that are acceptable both culturally and to families must become integral to the aims of assisted living. Programs promoting healthy habits and exercise, involvement of family members, Yoga, music therapy, and the use of technology are some of the interventions that are recommended to help address the prevalence of depression among AL residents. All health care workers of ALs in Jordan must be trained and qualified enough to recognize the symptoms of depression and how it could affect the health of older adults. They need to work in an interdisciplinary team, consisting of medical staff, occupational therapists, nurses, physical therapists, and other health professionals, to coordinate and collaborate on formulating a treatment plan to meet the residents' needs. The results of this study may act as a point of reference for the staff of AL facilities to initiate understanding and recognition of the symptoms of depression and related factors. 


\section{Acknowledgments}

This study was approved and funded by Deanship of Research at Jordan University of Science and Technology and Institutional Review Board of King Abdullah University Hospital/Irbid/Jordan.

\section{Disclosure}

The authors report no conflicts of interest in this work.

\section{References}

1. World Health Organization. Mental health and older adults. Available from: http://www.who.int/mediacentre/factsheets/fs381/en/. Accessed June 1, 2017.

2. Al-momani M, Almomani F, Alghadir AH, Gabr S. Factors related to gait and balance deficits in older adults. Clin Interv Aging. 2016;11: 1043-1049.

3. Hybels CF, Bennett JM, Landerman LR, Liang J, Plassman BL, Wu B. Trajectories of depressive symptoms and oral health outcomes in a community sample of older adults. Int J Geriatr Psychiatry. 2016; 31(1):83-91.

4. Watanabe $\mathrm{Y}$, Herano $\mathrm{H}$, Arai $\mathrm{H}$, et al. Relationships between frailty and oral health function in community dwelling elderly adults. $J$ Am Geriatr Soc. 2016;22(9):151-159.

5. Park SJ, Ko KD, Shin SI, Ha YJ, Kim GY, Kim HA. Association of oral health behaviors and status with depression, results from the Korean National Health and Nutrition Examination Survey, 2010. J Public Health Dent. 2014;74(2):127-138.

6. Cotten SR, Anderson WA, McCullough BM. Impact of internet use on loneliness and contact with others among older adults: cross-sectional analysis. J Med Internet Res. 2013;15(2):e39.

7. Pirhonen J, Pietilä I. Active and non-active agents: residents' agency in assisted living. Ageing Soc. 2016;11(2):1-18.

8. Allen J. Depression in assisted living. Geriatr Nurs. 2015;36(1):78-80.

9. Morgan LA, Perez R, Frankowski AC, Nemec M, Bennett CR. Mental illness in assisted living: challenges for quality of life and care. J Hous Elderly. 2016;30(2):185-198.

10. Gordon AL, Franklin M, Bradshaw L, Logan P, Elliott R, Gladman JR. Health status of UK care home residents: a cohort study. Age Ageing. 2014;43(1):97-103.

11. McLaren S, Turner J, Gomez R, McLachlan AJ, Gibbs PM. Housing type and depressive symptoms among older adults: a test of sense of belonging as a mediating and moderating variable. Aging Ment Health. 2013;17(8):1023-1029.

12. Nazemi L, Skoog I, Karlsson I, et al. Depression, prevalence and some risk factors in elderly assisted livingin Tehran, Iran. Iran J Public Health. 2013;42(6):559-569.

13. Kennedy D, Sylvia E, Bani-Issa W, Khater W, Forbes-Thompson S. Beyond the rhythm and routine: adjusting to life in assisted living. J Gerontol Nurs. 2005;31(1):17-23.

14. Hoover DR, Siegel M, Lucas J, et al. Depression in the first year of stay for elderly long-term nursing home residents in the USA. Int Psychogeriatr. 2010;22(7):1161-1171.

15. Samus QM, Onyike CU, Johnston D, et al. 12-Month incidence, prevalence, persistence, and treatment of mental disorders among individuals recently admitted to assisted living facilities in Maryland. Int Psychogeriatr. 2013;25(5):721-731.

16. Shahab S, Nicolici DF, Tang A, Katz P, Mah L. Depression predicts functional outcome in geriatric inpatient rehabilitation. Arch Phys Med Rehabil. 2017;98(3):500-507.

17. Chen X, Clark JJ, Chen H, Naorungroj S. Cognitive impairment, oral self-care function and dental caries severity in community-dwelling older adults. Gerodontology. 2015;32(1):53-61.
18. Harris Y. Depression as a risk factor for nursing home admission among older individuals. J Am Med Dir Assoc. 2007;8(1):14-20.

19. Suleiman K, Walter-Ginzburg A. A nursing home in Arab-Israeli society: targeting utilization in a changing social and economic environment. J Am Geriatr Soc. 2005;53(1):152-157.

20. Haugan G. Meaning-in-life in nursing-home patients: a correlate with physical and emotional symptoms. J Clin Nurs. 2014;23(7-8): 1030-1043.

21. Barca ML, Engedal K, Laks J, Selbaek G. A 12 months follow-up study of depression among nursing-home patients in Norway. J Affect Disord. 2010;120(1-3):141-148.

22. Jang Y, Park NS, Dominguez DD, Molinari V. Social engagement in older residents of assisted living facilities. Aging Ment Health. 2014; 18(5):642-647.

23. Al-Qudah H, Suliman S. Planning of nursing home care services in Jordan: its reality and challenges. Asian Soc Sci. 2011;7(10):94-101.

24. Khader F. Quality of life in the nursing homes in Jordan: perspectives of residents. Care Manag J. 2011;12(4):169-182.

25. Yesavage JA, Brink TL, Rose TL, et al. Development and validation of a geriatric depression screening scale: a preliminary report. J Psychiatr Res. 1983;17(1):37-49.

26. Pangman VC, Sloan J, Guse L. An examination of psychometric properties of the mini-mental state examination and the standardized mini-mental state examination: implications for clinical practice. Appl Nurs Res. 2000;13(4):209-213.

27. Tombaugh TN, McIntyre NJ. The mini-mental state examination: a comprehensive review. J Am Geriatr Soc. 1992;40(9):922-935.

28. Tinetti ME. Performance oriented assessment of mobility problems in elderly patients. J Am Geriatr Soc. 1986;34(2):119-126.

29. Alotaibi M. Cross-cultural adaptation process and pilot testing of the Arabic version of the Disability of the Arm, Shoulder and Hand (DASHArabic). Br Assoc Hand Ther. 2010;10:100-121.

30. Atieh M. Translation and validation of the Arabic version of the Geriatric Oral Health Assessment Index (GOHAI). Gerodontology. 2008; 25(1):34-41.

31. Almomani FM, McDowd JM, Bani-Issa W, Al-momani M. Healthrelated quality of life and physical, mental, and cognitive disabilities among nursing home residents in Jordan. Qual Life Res. 2014;23(1): $155-165$.

32. Al-Rajeh S, Ogunniyi A, Awada A, Daif A, Zaidan R. Preliminary assessment of an Arabic version of the Mini-Mental state examination. Ann Saudi Med. 1999;19(2):150-152.

33. Brislin RW. The Wording and Translation of Research Instruments. California: Sage Publications; 1986.

34. Kanwar A, Singh M, Lennon R, Ghanta K, McNallan SM, Roger VL. Frailty and health-related quality of life among residents of long-term care facilities. J Aging Health. 2013;25(5):792-802.

35. Rechardson J, Bedard M, Weaver B. Changing in physical functioning in institutionalized older adults. Disabil Rehabil. 2013;23(15):683-689.

36. Chen X, Clark JJ, Naorungroj S. Oral health in nursing home residents with different cognitive statuses. Gerodontology. 2013;30(1):49-60.

37. Sandhu NK, Kemp CL, Ball MM, Burgess EO, Perkins MM. Coming together and pulling apart: exploring the influence of functional status on co-resident relationships in assisted living. J Aging Stud. 2013; 27(4):317-329.

38. Cheng ST, Chow PK, Song YQ, et al. Mental and physical activities delay cognitive decline in older persons with dementia. Am J Geriatr Psychiatry. 2014;22(1):63-74.

39. Selbæk G, Engedal K, Bergh S. The prevalence and course of neuropsychiatric symptoms in nursing home patients with dementia: a systematic review. J Am Med Dir Assoc. 2013;14(3):161-169.

40. Resnick B. Interdisciplinary education and practice: where are we with all that? Geriatr Nurs. 2015;36(3):175-176.

41. Boyle A, Flores C. Assisted Living Administration and Management: Effective Practices and Model Programs in Elder Care. New York: Springer Publishing Company; 2010. 
42. Sewilam AM, Watson AM, Kassem AM, et al. Suggested avenues to reduce the stigma of mental illness in the Middle East. Int J Soc Psychiatry. 2015;61(2):111-120.

43. Almomani FM, Bani-Issa W. Physical, mental and cognitive disabilities in relation to utilization of dental care services by nursing home residents. Spec Care Dentist. 2017;37(3):126-133.

44. Smith M, Haedtke C. Depression treatment in assisted living settings: is an innovative approach feasible? Res Gerontol Nurs. 2013;6(2):98-106.

45. Mellor D, Davison T, McCabe M, George K. Personal careers' knowledge and response to depression among their aged-care clients: the care recipients' perspective. Aging Mental Health. 2008;12(3):389-399.

46. Fisher KA, Seow H, Brazil K, Freeman S, Smith TF, Guthrie DM. Prevalence and risk factors of depressive symptoms in a Canadian palliative home care population: a cross-sectional study. BMC Palliat Care. 2014; 13(1): 10 .
47. Atkins J, Naismith SL, Luscombe GM, Hickie IB. Psychological distress and quality of life in older persons: relative contributions of fixed and modifiable risk factors. BMC Psychiatry. 2013;13(1):249.

48. Zimmerman S, Dobbs D, Roth EG, Goldman S, Peeples AD, Wallace B. Promoting and protecting against stigma in assisted living and nursing homes. Gerontologist. 2016;56(3):535-547.

49. Szczepańska-Gieracha J, Kowalska J, Pawik M, Rymazewaska J. Evaluation of group based psychotherapy used as part of rehabilitation process in nursing home patients. Disabil Rehabil. 2014;36(12): 1027-1032.

50. De Boer ME, Leemriise CJ, Van Den Ende CH, Ribbe MW, Dekker J. The availability of allied health care by nursing homes. Disabil Rehabil. 2007;29(8):665-670.
Clinical Interventions in Aging

\section{Publish your work in this journal}

Clinical Interventions in Aging is an international, peer-reviewed journal focusing on evidence-based reports on the value or lack thereof of treatments intended to prevent or delay the onset of maladaptive correlates of aging in human beings. This journal is indexed on PubMed Central, MedLine,

\section{Dovepress}

CAS, Scopus and the Elsevier Bibliographic databases. The manuscript management system is completely online and includes a very quick and fair peer-review system, which is all easy to use. Visit http://www.dovepress. $\mathrm{com} /$ testimonials.php to read real quotes from published authors. 